\title{
Pickup and delivery problem with LIFO, time duration, and limited vehicle number
}

\author{
Andriansyah ${ }^{1,2^{*}}$, Nissa Prasanti $^{3}$, and Prima Denny Sentia ${ }^{1}$ \\ ${ }^{1}$ Laboratory of Industrial Computation and Optimization, University of Syiah Kuala, 23111 Aceh, \\ Indonesia \\ ${ }^{2}$ Industrial Engineering Department, University of Syiah Kuala, 23111 Aceh, Indonesia \\ ${ }^{3}$ Industrial Engineering Department, University of Teuku Umar, 23681 Aceh, Indonesia
}

\begin{abstract}
This study discusses pickup and delivery problem with last-infirst-out, time duration, and limited vehicle number which is usually experienced by door to door courier service. In this problem, company's vehicles will pick customer's goods and deliver them from one location to another. Technically, LIFO policy is enforced when the assigned vehicles for transporting the goods have a single access point to loading-unloading process. This study develops integer linear programming formulation based on three index formulation. The model is solved by branch-andbound algorithm. From experimental study, the result shows that optimal global solution, but takes long computation time. This computation time depends on the type of data used. From the 8 types of data with the same number of customers and vehicles, there is 1 data that can be solved within 1 second.
\end{abstract}

\section{Introduction}

Vehicle Routing Problem (VRP) is a problem in transportation that establish optimal routes and minimize transportation-related costs [1]. The minimum costs that can be minimize such as fuel consumption and balancing in a routes. Currently, there are many variants of VRP that have been developed, one of them is VRP with pickup and delivery service called Vehicle Routing Problem with Pickup and Delivery (VRPPD) or pickup and delivery problem (PDP). In PDP, the vehicle will visit the pickup location to pick the goods and directly deliver them to the delivery location. If the location visited by the vehicle has a range of service time, then it is called Pickup and Delivery Problem with Time Windows (PDPTW) [2]. In addition, according to [3], if the commodity transported is human, it is called Dial-a-Ride-Problem (DARP).

Many transportation companies related to PDP, such as courier service company. To increase competition, companies often propose some shipping packages, in order for fast shipment to the customer. To guarantee the shipment for the desired time, the company must make several policies, such as determining the duration of the vehicle's travel time. To reduce the cost associated with the vehicle, required an optimal route planning that consider the existing constraint. The general constraint in PDP are pairing and precedence constraint.

\footnotetext{
${ }^{*}$ Corresponding author: andriansyah@unsyiah.ac.id
} 
Pairing constraint is a constraint that items are picked up and delivered to the appropriate location, while precedence constraint is a constraint that the vehicle will pick the goods first before delivered to the corresponding location. For vehicles that have only one access door for loading and unloading and transport dangerous goods, heavy goods and fragile goods must apply a last-in-first-out (LIFO) rule to facilitate the unload process at the delivery location [4]. This variant is called pickup and delivery problem with LIFO and time duration (PDPLT) [5].

In theory, PDP has an assumption of an unlimited number of vehicles or all customers can be served, for example in paper [5]-[7]. In practice, with a limited number of vehicles, not all customers can be served. Therefore, there should be optimal planning to minimize the number of unserved customer. The contribution of this paper is developing a formulation for PDPLT with a limited number of vehicles, so the objective function of this paper is minimizing the total travel time of the vehicle and the number of unserved customer. In this paper, the variant is called pickup and delivery problem with LIFO, time duration, and limited vehicle or PDPLTV.

\section{Literature Review}

Based on the existing literature, there are so many variants of VRP that have been developed. VRP is a problem of k-TSP where TSP has not considered the capacity of the vehicle. For TSP, the salesman is assigned to serve all customer's requests with the restrictions of all customers served only once. TSP problems often occur with two types of services: pick-up and delivery, abbreviated as TSPPD [8].

The problem of PDP is a generalization of the TSPPD problem that consider the capacity of the vehicle. Now, PDP problems approach to real conditions and bring new variants, for example every customer has a varied service time or PDPTW [2], each vehicle has a travel duration and must follow the LIFO rules (PDPLT) [5]. [2] developed a threeindex mathematical formulation for PDPTW then solved with a column generation algorithm. [9] apply the soft time windows for PDPTW then solved it with reactive tabu search algorithm. [10] solving the same problem using a construction novel heuristic and then improve it using tabu search algorithm. [11] developed the tabu search-simulated annealing algorithm and successfully solved the problem on a large scale. Genetic algorithm implementation for PDPTW was first conducted in the study [12]. [13] solved the same problem using a column generation algorithm but considering heterogeneous vehicles. [14] developed a mathematical formulation for PDPTW. The developed mathematical formulation is a two-index based formulation then solved it using a branch and cut algorithm. [15] solved the PDPTW using ALNS algorithm. [16] developed a two-stage simulated annealing and LNS algorithm for similar problems. For a literature study on PDPTW, this study recommends reading a survey conducted by [17]. Surveys are classifying the types of problems pickup and delivery of goods such as many-to-many, many-to-one, and one-to-one. In addition, this study also recommends surveys conducted by [18] and [19].

The LIFO rule of pick-up and delivery arises from handling charges if the goods transported by the vehicle are dangerous, very heavy, and fragile goods. The appearance of this constraint is technical, when the vehicle performs the unloading process. The used assumption for this problem is the used vehicle to transport the goods only has one access loading-unloading. The concept of LIFO was first investigated by Cassani (2004) in [20] in the TSPPD. [21] developed the VNS algorithm to solve similar problem. Then in the [21] developed a mathematical model and exact algorithm for this problem. [22] developed a branch and cut algorithm for similar problem. [23] developed a mathematical model for this problem and implemented this rule in TSPPD. However, in that model, LIFO rules may be 
violated with a record of additional handling fees. [24] developed a tree representation and cut branch algorithm for this problem. [25] solved the same problem, but considering the maximum distance limit for vehicles. [26] developed a metaheuristic algorithm for the TSPPD with handling cost that successfully solved for the existing data using local search and tabu search. The most recent case considering LIFO was examined by [7] who developed a mathematical model for PDPTW with LIFO constraints based to three index models [27], then solved it using branch and cut and price algorithms. The following year [20] developed the GA-LS algorithm to solve large-scale cases and successfully implemented.

\section{Model Formulation}

PDPLTV is a generalization of the Benavent's PDPLT model [5]. In this model, the number of vehicles is limited, so in practice, not all customers will be served by vehicles. The aim of this problem is to minimize the total travel time and minimize the number of unserved customers. Each solution should be feasible where it does not violate the duration time of vehicle, vehicle capacity, precedence, pairing, LIFO, and the number of vehicles used. In this case a network $G$ consisting of a set of nodes $N$ and a set of $\operatorname{arcs} A . N=O U$ $P \cup D$ which $O=\{0,2 n+1\}$ is depot, $P=\{1,2, \ldots, n\}$ is pickup and $D=\{n+1, \ldots, 2 n\}$ is delivery. Each nodes $i \in P$ has a request $q_{i}>0$ that should be sent to $(i+n) \in D$ or request $q_{i+n}=-q_{i}$. Each point $i \in O$ has opening time $e_{i}$ and closing time $l_{i}$. The opening time and closing time are also used as the maximum duration that the vehicle must depart from the depot and return to the depot. Each link $(i, j) \in A$ is associated with travel time $t_{i j}$. Then a vehicle set $K=\{1,2, \ldots, v\}$ is also used in this problem. Each $k \in K$ has a vehicle capacity $Q$. To formulate the problem into integer linear programming (ILP) model, it defines several decision variables. $L_{i}^{k}$ and $T_{i}^{k}$ is a non-negative continuous decision variable and is related to vehicle load and vehicle travel time. $x_{i j}^{k}$ is a decision variable. 1 if the vehicle travels and 0 to vice versa. PDPLTV is represented in a three-index integer linear programming. The formulations are based on [6] and [7]. The ILP model of the problem is as follows:

$$
\begin{gathered}
\operatorname{minimize} Z=\alpha\left(\sum_{k \in K} \sum_{i \in N} \sum_{j \in N} t_{i j} x_{i j}^{k}\right) / 60+\beta\left(\left(n-\left(\sum_{k \in K} \sum_{i \in N} \sum_{j \in N} x_{i j}^{k}-v\right)\right) / 2\right) \\
T_{j}^{k} \geq T_{i}^{k}+t_{i j}-M\left(1-x_{i j}^{k}\right) ; \forall k \in K, i \in N, j \in N \\
L_{j}^{k} \geq L_{i}^{k}+q_{j}-M\left(1-x_{i j}^{k}\right) ; \forall k \in K, i \in N, j \in N \\
e_{i} \leq T_{i}^{k} \leq l_{i} ; \forall k \in K, i \in O \\
x_{i j}^{k}=0 ; \forall k \in K, i \in N, j \in N \backslash\{i\} \\
\sum_{k \in K} x_{i j}^{k}=0 ; \forall i \in N ; \forall j \in N \\
L_{i}^{k}<=q_{i} ; \forall i \in N, \forall k \in K \\
\sum_{j \in N} x_{k j}^{k}=1 ; \forall k \in K \\
\sum_{j \in N} x_{j i}^{k}-x_{i j}^{k}=0 ; \forall k \in K, i \in N \\
\sum_{k \in K} \sum_{j \in N} x_{k j}^{k} \leq v
\end{gathered}
$$




$$
\begin{gathered}
\sum_{k \in K} \sum_{j \in N} x_{i j}^{k} \leq 1 ; \forall i \in N \\
\sum_{j \in N} x_{i j}^{k}-\sum_{j \in N} x_{j(i+n)}^{k}=0 ; \forall k \in K, \forall i \in P \\
T_{i}^{k}+t_{i(i+n)} \leq T_{i+n}^{k} ; \forall k \in K, \forall i \in P \\
L_{(n+i)}^{k}=L_{i}^{k}-q_{i} ; \forall k \in K, \forall i \in P \\
x_{i j}^{k}=0 ; k \in K, i \in P, j \in D \backslash\{i+n\} \\
L_{i}^{k} \geq 0 ; \forall k \in K, \forall i \in N \\
T_{i}^{k} \geq 0 ; \forall k \in K, \forall i \in N \\
x_{i j}^{k} \in\{0,1\} ; \forall k \in K, i \in N, j \in N
\end{gathered}
$$

The objective function is to minimize the travel time and the number of unserved customers can be seen in the formulation (1). Inequalities (2) and (3) represent the consistency of time and vehicle load variables with $\mathrm{M}$ is a big number. Maximum duration of time is represented in equation (4). Equations (5) and (6) to prevent the occurrence of subtour in the solution. The constraint of vehicle capacity can be seen in (7). Equation (8) denotes that the vehicle departs and returns to the depot. The vehicle will leave the customer right after serve the customer can be seen in equation (9). Equation (10) is a constraint of the number of vehicles used does not exceed the number of vehicles available. The customer is served by a vehicle at least once on the constraint (11). Equations (12) and (13) are pairing and precedence constraint. The LIFO constraint can be seen in (14) and (15). constraint (16) and (17) are non-negative. The decision variable can be seen in (18).

\section{Computational Study}

The computational experiment is performed on Intel ${ }^{\circledR}$ Core ${ }^{\mathrm{TM}}$ i5-3470 $3.20 \mathrm{GHz}$ (4CPUs) 4 GB RAM, under Windows 10 operating system. LINGO was used for solving small instances. The algorithm used in LINGO is branch and bound.

Table 1. Computational results.

\begin{tabular}{cccccc}
\hline No & Instance & Objective & Unserved Customer & Solution & Time (s) \\
\hline 1 & 1c101_20_0 & 251,33 & 2 & Optimal global & 121 \\
2 & 1c102_20_0 & 363,33 & 4 & Optimal global & 249 \\
3 & 1c103_20_0 & 353,33 & 4 & Optimal global & 18 \\
4 & 1c104_20_0 & 379,33 & 5 & Optimal global & 1 \\
5 & 1c105_20_0 & 349,33 & 4 & Optimal global & 5 \\
6 & 1c106_20_0 & 243,33 & 2 & Optimal global & 182 \\
7 & 1c107_20_0 & 368,67 & 5 & Optimal global & 6 \\
8 & 1c108_20_0 & 371,33 & 4 & Optimal global & 815 \\
\hline
\end{tabular}

The test instances used in this experiment was generate by [5]. The passenger and package request randomly generated the following uniform distributions. Each request has pickup and delivery nodes. The difference of this study with [5] lies in the number of vehicles 
used. In this study, the number of vehicles used is limited so there are some unserved customers while [5] still assumes all customers must be served. this can be seen from the objective function of the model. We make a little modification of this data in accordance with the needs of PDPLTV. Parameter $\alpha=40$ and $\beta=60, v=2, n=7$ are used in this experiment. Solutions depend on the selection of $\alpha$ and $\beta$ values. These values are a priority of the objective function. if the alpha is too large, the number of unserved customers is increasing, but if it is too small, it will increase the cost. All tested instances from Table 1, the result solution is feasible and global optimal. From the results, the computation time shown is relatively long, because, the developed formulation is three index, so to find the optimal solution, takes a very long time, especially the algorithm used is an exact algorithm.

\section{Conclusions and Future Work}

This study proposed the PDPLTV model to address the problems on courier service. The objective of this model is to minimize total travel time and number of unserved customer. The contribution of this paper is consider limited vehicle number and LIFO rule. Results indicated that the three index formulation model generate optimal global for small instance. The limitations of this approach are very difficult to generate and the use long computation, but will produce a very good solution. Future research could apply the model and the proposed metaheuristic algorithm for solving bigger instances and compare the results.

\section{References}

1. P. Toth and D. Vigo, Vehicle Routing Problem, Methods, and Application, 2nd ed., Philadelphia, SIAM, (2014)

2. Y. Dumas, J. Desrosiers, and F. Soumis, Eur. J. Oper. Res., 54, 1 (1991)

3. J. F. Cordeau and G. Laporte, The Dial-a-Ride Problem (DARP): Variants, modeling issues and algorithms, 4or, vol. 1, no. 2, pp. 89-101, (2003)

4. F. Carrabs, J.-F. Cordeau, and G. Laporte, INFORMS J. Comput., 19, 4 (2007)

5. E. Benavent, M. Landete, E. Mota, and G. Tirado, Eur. J. Oper. Res., 243, 3 (2015).

6. M. G. S. Furtado, P. Munari, and R. Morabito, Pickup and delivery problem with time windows: A new compact two-index formulation, Oper. Res. Lett., vol. 45, no. 4, pp. 334-341, (2017)

7. M. Cherkesly, G. Desaulniers, and G. Laporte, Transp. Sci. Artic. Adv., 1-15 (2014)

8. B. Kalantari, A. V. Hill, and S. R. Arora, Eur. J. Oper. Res., 22, 3 (1985)

9. W. P. Nanry and J. Wesley Barnes, Transp. Res. Part B Methodol., 34, 2, (2000)

10. H. C. Lau and Z. Liang, IEEE Comput. Soc. eds, 13th IEEE Int. Conf. Tools with Artif. Intell., pp. 333-340 (2001)

11. H. Li and A. Lim, Proc. 13th IEEE Int. Conf. Tools with Artif. Intell. ICTAI 2001, pp. 160-167 (2001)

12. W. Jih, C. Kao, and J. Y. Hsu, Proc. 2002 IEEE Int. Symp. Intell. Control, 496-501 (2002)

13. H. Xu, Z.-L. Chen, S. Rajagopal, and S. Arunapuram, Transp. Sci., 37, 3 (2003)

14. Q. Lu and M. Dessouky, Transp. Sci., 38, 4, 503-514 (2004)

15. D. Pisinger and S. Ropke, Transp. Sci., 40, 455-472, (2006)

16. R. Bent and P. Van Hentenryck, Comput. Oper. Res., 33, 4 (2006)

17. G. Berbeglia, J.-F. Cordeau, I. Gribkovskaia, and G. Laporte, 15, 1 (2017)

18. S. N. Parragh, K. F. Doerner, and R. F. Hartl, J. für Betriebswirtschaft, 58, 2 (2008)

19. S. N. Parragh, K. F. Doerner, and R. F. Hartl, J. für Betriebswirtschaft, 58, 2, (2008) 
20. M. Cherkesly, G. Desaulniers, and G. Laporte, Comput. Oper. Res., 62, 23-35 (2015)

21. F. Carrabs, R. Cerulli, and J.-F. Cordeau, INFOR Inf. Syst. Oper. Res., 45, 223-238 (2008)

22. J.-F. Cordeau, M. Iori, G. Laporte, and J. J. Salazar González, Networks, 55, 1 (2010)

23. M. Battarra, G. Erdoğan, G. Laporte, and D. Vigo, Transp. Sci., 44, 3 (2010)

24. Y. Li, A. Lim, W. C. Oon, H. Qin, and D. Tu, Eur. J. Oper. Res., 212, 3 (2011)

25. B. Cheang, X. Gao, A. Lim, H. Qin, and W. Zhu, Eur. J. Oper. Res., 223, 1, (2012)

26. G. Erdoğan, M. Battarra, G. Laporte, and D. Vigo, Comput. Oper. Res., 39, 5, (2012)

27. S. Ropke, J.-F. Cordeau, and G. Laporte, Networks, 49, 258-272 (2007) 